\title{
10 Ethische Konflikte bei Kindernotfällen
}

\author{
Thomas M. Boesing, Tanja Löbbing, Bernhard Kretschmer, \\ Eckard Hamelmann und Klaus Kobert
}

\subsection{Einführung}

Ethische Konflikte bei Notfällen im Kindesalter sind bislang wenig untersucht worden. Die Autoren berichten daher in erster Linie aufgrund ihrer langjährigen Erfahrung als Kinderärzte und als Klinische Ethiker.

Notfälle können inner- und außerhalb der Klinik vorkommen. Während sie außerhalb der Klinik meistens unvorhersehbar eintreten, ist bei schweren stationären Krankheitsverläufen, beispielweise auf der Intensivstation, eine Notsituation häufig antizipierbar und Bestandteil des Behandlungsverlaufs. Darin begründet sich als wesentlicher Unterschied der beiden Szenarien die vorhandene bzw. fehlende Möglichkeit, vorbereitende Absprachen für den Notfall zu treffen.

\subsection{Außerklinische Notfälle}

Der Notarzt wird in der Regel zu einem akut eingetretenen Ereignis gerufen, welches zuvor nicht absehbar war. Typische Beispiele sind Verkehrs-, Verbrühungs- und Ertrinkungsunfälle sowie Vergiftungen und hochakute Erkrankungen wie Fieberkrämpfe oder Septikämien. Gelegentlich wird er vielleicht auch eine Geburt betreuen müssen oder zu einem plötzlichen Kindstod gerufen. Im notfallmedizinischen Kontext muss immer zumindest eine medizinische Screeninguntersuchung erfolgen, die auch integraler Bestandteil in den Versorgungsalgorithmen der Reanimationsabläufe des Advanced Trauma Life Support (ATLS) und des European Pediatric Life 
Support (EPLS) ist. Im besten Interesse des Kindes erfolgt dann, gegebenenfalls auch ohne elterliche Einwilligung, jede lebenswichtige oder nicht ohne wesentlichen Schaden für den Patienten aufschiebbare Therapie. Diese darf keinesfalls vorenthalten oder verzögert werden, nur weil die elterliche Zustimmung nicht eingeholt werden kann. Jede weitere nicht unmittelbar lebenserhaltende Diagnostik und Therapie bedarf dann aber zwingend einer elterlichen Aufklärung und Zustimmung oder deren Ersetzung durch einen familiengerichtlich bestellten Ergänzungspfleger oder Vormund.

Bei der Durchführung risikoreicher Maßnahmen in der Notfallversorgung erwachsener Patienten, wie beispielsweise der endotrachealen Intubation, ist der darin unerfahrene Notarzt eher zurückhaltend (Adams u. Flemming 2015). Bei der Versorgung von Kindern bekommt dieser Aspekt wahrscheinlich eine umso größere praktische Relevanz, da viele Notärzte in der Behandlung von Kleinkindern und Säuglingen auch in der Durchführung von Basismaßnahmen, wie der Anlage eines venösen Zugangs, unerfahren sind. Dieses Kompetenzdefizit kann in solchen Fällen zu nicht-adäquaten Handlungen bei der Erstversorgung führen .

Der pädiatrisch unerfahrene Notarzt sieht sich in jedem Fall mit einer ihn belastenden Situation konfrontiert. Er muss unter Zeitdruck zwischen den aktuellen medizinischen Bedürfnissen des Kindes und seinen persönlichen Fähigkeiten abwägen, um so den Weg des geringsten Schadens bei gleichzeitig maximal durch ihn persönlich erreichbarem Nutzen zu beschreiten.

Dieses Dilemma lässt sich vor Ort selten auflösen, da weder ein erfahrener Kollege zeitnah hinzugezogen, noch der Kompetenzzuwachs kurzfristig erreicht werden kann. Daraus können erhebliche psychische Belastungen für den Notarzt resultieren.

Verstärkt wird die Situation durch die Anwesenheit naher Patientenangehöriger, die ihr Kind einer akuten Bedrohung ausgesetzt sehen. Nicht immer sind zu ihrer Betreuung hinreichende personelle Ressourcen unmittelbar verfügbar. Die Versorgung des Kindes selbst bindet, schon wegen der Seltenheit dieser kindlichen Notsituationen, meist alle vorhandenen Rettungskräfte.

Im unvorhergesehenen Notfall besteht immer zunächst eine Verpflichtung zum Lebenserhalt.

\subsection{Innerklinische Notfälle}

Bei Kindern, die sich in stationärer Behandlung befinden, sind die selten auftretenden schwerwiegenden und vital bedrohlichen Notfallsituationen häufig vorhersehbar. Kritische Lebenslagen, die mit einer Atem- oder Kreislaufinsuffizienz einhergehen, bahnen sich im Verlauf einer Erkrankung meistens an. Hier sind insbesondere Intensivpatienten zu nennen, bei denen nach längerer Therapie lebensgefährliche Komplikationen auftreten, wie z.B. schwere Septikämien. Hier kann und sollte aber im Vorfeld eine strukturierte Entscheidungsfindung durch geeignete Verfahren erfolgen:

In regelmäßig durchgeführten Ethik-Visiten werden Fragestellungen zu einzelnen Patienten im Team zusammen mit einem Klinischen Ethiker erörtert (Kobert u. Löb- 
bing 2013). Unter Berücksichtigung von Prognose, Indikationsstellung und Elternpositionen werden Anregungen und Empfehlungen erarbeitet, die durch die juristisch Verantwortlichen, nämlich den behandelnden Arzt und die Erziehungsberechtigten, in Anordnungen umgesetzt und schriftlich fixiert werden sollten. Diese legen dann für den Notfall Therapieziele und -einschränkungen, wie Anordnungen eines Reanimationsverzichts oder bestimmter Medikationen, fest. Derartige Ethik-Visiten werden im Ev. Krankenhaus Bielefeld zunehmend von unterschiedlichen Stationen nachgefragt, so auch von der Pädiatrischen Intensivstation. Dort wurden im Jahr 2014 an 16 Terminen Ethik-Visiten durchgeführt; dabei wurden 29 Fälle besprochen. Vorrangig ging es um das Therapieziel (41,4\%; 12 von 29$)$ und um die konkrete Ausgestaltung der Behandlung (20,7\%; 6 von 29). Empfohlen wurde in 17,2\% der besprochenen Fälle ein palliatives Vorgehen ( 5 von 29), in 10,3\% (3 von 29) sollte zunächst der Verlauf abgewartet bzw. die Therapie auf gleichbleibendem Niveau fortgesetzt $(6,9 \%$, 2 von 29) werden. Die Aufnahme einer Maximaltherapie sowie weitere Kommunikation mit Eltern und Behandlungsteam waren in je 6,9\% (2 von 29) die im Rahmen einer ethischen Visite empfohlene Vorgehensweise.

Zusätzlich zu diesen für das gesamte Kalenderjahr im Vorhinein festgelegten Visitenterminen finden bei Bedarf auf Anforderung kurzfristig anberaumte Ethik-Fallgespräche zu einem konkreten Kind statt (Kessler-Weinrich 2015). Von Januar 2006 bis einschließlich Dezember 2014 fanden 58 Ethik-Fallgespräche im Kinderzentrum statt. Sie werden ebenfalls in multidisziplinärer Besetzung durchgeführt, die Teilnahme der Eltern ist bei diesem Format der Ethikberatung ausdrücklich gewünscht. In dem benannten Zeitraum nahmen in über einem Drittel der Gespräche (37,9\%; 22 von 58 ) Eltern daran teil. Die Fallgespräche dauern im Mittel etwas mehr als eine Stunde (64 \pm 25 Min.) und werden ausführlich protokolliert. Resultierende Anordnungen bezüglich der weiteren Behandlung werden routinemäßig auf dem Formular „Empfehlungen zum Vorgehen in Notfallsituationen“ (Rellensmann u. Hasan 2009) getroffen. Dadurch werden Transparenz und Informationsweitergabe gefördert. Diese Praxis der vergangenen zehn Jahre hat gezeigt, dass diese Vorgehensweise in mehrfacher Hinsicht hilfreich ist. Ihre Evaluation ergab, dass diese Art der Entscheidungsfindung in den allermeisten Fällen von den Mitarbeitenden im Nachhinein als für das Kind angemessen und für das Team hilfreich eingestuft wird. Die Empfehlungen der Fallgespräche wurden in der Regel umgesetzt.

Die Unterscheidung zwischen präklinischem und klinischem Notfall ist nur grob orientierend und richtungsweisend. Selbstverständlich trifft auch ein Notarzt auf Kinder mit einem schweren langjährigen Verlauf einer lebenslimitierenden Erkrankung. Hier wäre, wie im Krankenhaus, vorab eine differenzierte Entscheidungsfindung bezüglich der Therapie mit entsprechender Dokumentation als Richtschnur für den Notarzt angezeigt.

Gleichwohl kann in der Klinik eine Notsituation völlig unvorhergesehen entstehen, sodass auch hier zunächst die Verpflichtung zum Lebenserhalt durch therapeutisches Handeln besteht. Eine sorgfältige Abwägung, ob dieses Vorgehen zum Wohle des Kindes war, ist hier, wie auch in der präklinischen Situation, erst im Nachhinein möglich. Sollte sich dabei zeigen, dass diese Prämisse nicht mehr zutrifft, weil die begonnene Behandlung beispielsweise nicht mehr indiziert ist, müssen die eingeleiteten Schritte zurückgenommen werden. 


\section{Ethik-Visiten und Ethik-Fallgespräche können in schwierigen Situationen für alle Beteiligten Hilfe und Unterstützung bieten. \\ Bei absehbaren gesundheitlichen Krisen sollte mit den Mitteln der strukturierten ethischen Entscheidungsfindung wie Ethik-Visiten und Ethik-Fallgesprächen das therapeutische Vorgehen in verantwortungsvoller Weise abgestimmt werden.}

\subsection{Pädiatrische Entwicklungsphasen}

Patienten jeden Alters begegnen Ärzte und Pflegende mit dem Wunsch, ihnen therapeutisch zu helfen (Benefizienz), ihnen nicht zu schaden (Nonmalefizienz), die Behandlungsmöglichkeiten unter ihnen gerecht zu verteilen (Gerechtigkeit) und ihnen auch zur Seite zu stehen (Fürsorge und Würde), besonders dann, wenn die Behandlungsteams zu einer Heilung nicht mehr verhelfen können (DAKJ 2007).

Einzig der Aspekt der Patientenautonomie (Beauchamp u. Childress 2012) aber ist in der Pädiatrie entsprechend des individuellen Entwicklungsstands des Patienten differenziert zu betrachten. So werden zunächst die Entwicklungsphasen eines Kindes hin zu einem Erwachsenen beleuchtet, um die Voraussetzungen für eine autonome Entscheidung zu verstehen.

Die kindliche Entwicklung kann in ihren verschiedenen Phasen häufig interpersonell vergleichbar, aber oft auch mit sehr hoher Variabilität verlaufen. Daran angepasst muss es deshalb eine individuelle entwicklungsentsprechende Prüfung der Einwilligungsfähigkeit als Voraussetzung zur Wahrnehmung des Selbstbestimmungsrechts bei ethischen Fragestellungen im Kindesalter geben. Während ein Neugeborenes und auch ein Säugling noch völlig abhängig von elterlicher Betreuung sind und die Eltern auch im weiteren Kindesalter eine stellvertretende Entscheidungsfindung vornehmen, wird der Jugendliche, aber auch das mit seinem chronischen Erkrankungsbild schon seit Jahren vertraute Schulkind, ein hohes Maß an selbstbestimmter Entscheidung (Autonomie) mittragen wollen und können.

Als Neugeborenes verfügen wir über Flucht- und Schutzreflexe, trinken und schlafen. Der Wahrnehmungshorizont wächst im ersten Lebensjahr immens. Mit etwa acht Wochen belohnen wir unsere Eltern mit dem ersten sozialen Lächeln und erkunden dann von Monat zu Monat mit wachsenden motorischen Kompetenzen zunehmend unsere Umwelt. Der Säugling verdreifacht im ersten Lebensjahr sein Körpergewicht, die Proportionen ändern sich auch in den folgenden Jahren erheblich. Von den früh beobachtbaren bewussten Handlungen des Säuglings (o-1 Jahre) hin zum Erkennen einfacher Zusammenhänge schon beim Kleinkind (1-3 Jahre), kann das Kindergartenkind (3-5 Jahre) mit seinen „Warum“-Fragen bereits konkrete Kausalitäten erfassen. Parallel zur schulischen Entwicklung entfaltet sich, ausgehend von der zunächst sehr egozentrischen Wahrnehmung des Kleinkindes, eine wachsende Denkfähigkeit, die bereits dem Grundschulkind (6-9 Jahre) ein „Grund-Folge-Denken“ ermöglicht. Ein älteres Schulkind (10-13 Jahre) und mehr dann auch ein Jugendlicher (14-17 Jahre) verfügt mit seinem zunehmenden Abstraktionsvermögen in aller Regel schon über eine gute Einsichtsfähigkeit in grundlegende medizinische Sachverhalte, sodass hier von einer zunehmenden Mitsprache- und Selbstbestimmungskompetenz in medizinischen Belangen auszugehen ist. 
Deshalb flacht etwa nach dem 14. Lebensjahr bis zum Zeitpunkt der vollen „Heranreifung" das dreieckige Beziehungsgeflecht, in dem bis dahin in der Pädiatrie zwischen dem Patienten und dem Arzt noch die Eltern als Träger der Entscheidung stehen, immer mehr zu einer zweiseitigen Behandlungsbeziehung ab. Der autonome Jugendliche hat damit auch ein Recht auf volle Aufklärung über seine gesundheitliche Situation auch gegen den Wunsch seiner Eltern, wie es in manchen palliativen Therapiesituationen angetroffen wird. Wann letztlich eine volle Einsichtsfähigkeit gegeben ist, ist nach der Auffassung des Bundesgerichtshofes nicht so sehr eine Frage des erreichten nummerischen Alters, als vielmehr eine Frage der „geistigen und sittlichen Reifung“ (grundlegend BCH NJW 1959, 811). Ist diese gegeben, dann erreicht der Jugendliche die volle Einwilligungsfähigkeit in allen medizinischen Belangen bereits vor Erreichen seiner vollen Geschäftsfähigkeit mit 18 Jahren. Er kann dann unter Umständen sogar eine Schweigepflicht des Arztes gegenüber seinen Eltern einfordern.

Auf den Heranwachsenden (18-20 Jahre) wiederum kann im Falle einer Straffälligkeit bei einem erkennbaren Reifungsdefizit noch das mildere Jugendstrafrecht angewendet werden. Das hat aber für die Frage der vollen Einwilligungsfähigkeit des Heranwachsenden keine Bedeutung.

\subsection{Autonomie bei Kindern und Jugendlichen}

Da jede ärztliche Handlung, die mit einem Eingriff in die körperliche Integrität verbunden ist, formaljuristisch den Tatbestand einer Körperverletzung erfüllt, erhält sie erst durch die Einwilligung nach sachgerechter Aufklärung ihre Legitimation. Hauptakteure der Einwilligung sind in der Pädiatrie in aller Regel die Eltern, bei schweren Eingriffen sogar zwingend beide Elternteile. Nach der Dreistufentheorie werden Routinefälle, bei denen die Einwilligung eines begleitenden Elternteils ausreichend ist, von mittelschweren Eingriffen mit „nicht unbedeutenden Risiken“ unterschieden, bei denen die Verpflichtung des Arztes besteht, nach der Autorisierung des erschienenen Elternteils hinsichtlich der gemeinsam erforderlichen Zustimmung zu diesem Eingriff zu fragen. Bei außergewöhnlich schwierigen Eingriffen muss sich der Arzt Gewissheit verschaffen, dass der nicht erschienene Elternteil mit der Vornahme des Eingriffs einverstanden ist (Harder 2004) und dies auch dokumentieren.

Alle ethischen Prinzipien gelten auch für Kinder und Jugendliche. Deren wachsender Autonomie ist Rechnung zu tragen, da die Selbstbestimmungsfähigkeit zumeist vor der Volljährigkeit erreicht wird.

\subsubsection{Die Frage nach der Einwilligungsfähigkeit bei Kindern und Jugendlichen}

Bei ethischen Entscheidungsfindungen sind die Präferenzen des Patienten bezüglich einer Behandlungsoption von herausragender Bedeutung. Medizinische Maßnahmen dürfen nicht gegen den Willen eines einwilligungsfähigen Menschen durchgeführt werden. Dem Respekt vor der Patientenautonomie eines voll urteilsfähigen Erwachsenen kommt eine besondere, den anderen Prinzipien, insbesondere auch dem der Benefizienz vorgeordnete Rolle $\mathrm{zu}$. Als Kennzeichen einer selbstbestimmten Ent- 
scheidung, beispielsweise zur Erlangung eines „informed consent“, muss also eine hinreichende bereichsbezogene Urteilsfähigkeit nach vollumfänglicher Aufklärung gefordert sein, bei der auch alle Aspekte und Konsequenzen einer Behandlung oder Nichtbehandlung verstanden worden sein müssen.

Jugendliche sollten entsprechend ihres Reifezustandes aufgeklärt werden, wobei sie bezüglich einer konkreten Therapie durchaus einwilligungsfähig sein können. Der Frage nach der Einwilligungsfähigkeit dieser Patienten kommt somit eine hohe Bedeutung zu. Immerhin kann sich der autonom entscheidende Erwachsene mit voller Kompetenz und Urteilsfähigkeit nach erfolgter ausführlicher Aufklärung, die alle Konsequenzen eines Für und Wider einer Entscheidung mit berücksichtigt, sogar gegen eine klar indizierte medizinische Maßnahme entscheiden. Allerdings setzt insbesondere die Ablehnung einer medizinisch indizierten Maßnahme ein weit höheres Urteilsvermögen voraus als die Einwilligung (Harder 2004). Hier liegt aber die Grenze der Entscheidungsfähigkeit für die autonome Entscheidung eines Jugendlichen (Royal College of Paediatrics and Child Health 2004). Der Arzt sollte sich in diesem Fall besonders bemühen, die Eltern in den Entscheidungsprozess miteinzubeziehen, um auch mit diesen eine konsensuelle Entscheidung herbeizuführen.

Konsensuelle Entscheidungsfindung bleibt auch im Dreiecksverhältnis zwischen Patient, Eltern und Arzt im wohlverstandenen Interesse des Kindes ein wichtiges Ziel.

Dem Jugendlichen kommt also ein stufenweises Mitspracherecht zu. Als Anhalte für eine hinreichende sittliche Reife zu einer autonomen Entscheidungsfähigkeit können dabei insbesondere gelten, dass der Patient bereits im eigenen Haushalt wohnt, schon verheiratet ist oder bereits eigene Kinder hat (American Academy of Pediatrics $[\mathrm{AAP}]$ 2011)

Es gibt sicher eine unterschiedliche Einsichtsfähigkeit des einzelnen Jugendlichen zu unterschiedlichen Fragestellungen. Die Erkundung und Beurteilung der Einsichtsfähigkeit des unmündigen Patienten ist dabei Aufgabe des Arztes und muss in jedem Einzelfall von diesem dokumentiert werden. Es ist wenig hilfreich, einen nicht einsichtsfähigen Patienten nach der „Erlaubnis“ zu einer medizinischen Maßnahme zu fragen, wenn bei einer zu erwartenden Ablehnung nicht auf diesen Wunsch eingegangen werden kann. Was wird das Grundschulkind antworten, wenn bei ihm die Durchführung einer Lumbalpunktion geplant ist?

Obwohl in solchen Situationen der natürliche Wille nicht zur Leitschnur des Handelns gemacht werden kann, müssen die Behandelnden auch diesem Kind an seine Einsichtsfähigkeit angepasst die erforderlichen Maßnahmen erklären, um möglichst eine Duldung (,,informed assent“) zu erreichen (Steurer u. Berger 2012). Für die klinische Prüfung von Arzneimitteln und Medizinprodukten bedarf es schon bei Schulkindern auch deren Einwilligung (neben jener der Eltern), was eine altersgerechte Aufklärung verlangt.

Dem Grad der Entscheidungsfähigkeit bei Kindern und Jugendlichen kommt eine zentrale Bedeutung zu. 


\subsubsection{Der Umgang mit der Schweigepflicht und das Wohl des Kindes}

Die Schweigepflicht des Arztes kommt auch gegenüber den Eltern eines einsichtsfähigen Patienten zum Tragen, so z.B. typischerweise für die Behandlung von mit Schwangerschaft assoziierten Erkrankungen, Antikonzeption, Drogenerkrankungen, sexuell übertragener Erkrankungen und auch psychischer Probleme.

In den seltenen Fällen eines nicht auszuräumenden Dissenses zwischen den Eltern und dem behandelnden Arzt besteht für diesen unter Wahrung der Verhältnismäßigkeit bei einem objektiv erkennbaren Verstoß gegen die Sorgepflicht der Eltern nicht nur ein Behandlungsrecht, sondern vielmehr eine eigenständige Behandlungspflicht. Bestehen die Sorgeberechtigten auf die Realisierung von Vorstellungen, die einen groben Verstoß gegen das Wohl des Kindes bedeuten, muss der Arzt auf eine familiengerichtliche Entscheidung hinwirken (Dierks et al. 1995). Eltern sind aber auch im Unterschied zu Betreuern in ihrer Entscheidung, z. B. in religiösen und weltanschaulichen Belangen, so lange nicht an den vermeintlichen Willen des Patienten - ihres Kindes - gebunden, wie sie nicht offenkundig gegen das „Kindeswohl“ verstoßen. Denn dieses Kindeswohl zu interpretieren, obliegt zunächst einmal ihrem Recht und ihrer Pflicht ( $\$ 1626$ BCB, Art. 6 GC), wobei sie auf die wachsende Selbstbestimmungsfähigkeit ihres Kindes Rücksicht nehmen müssen ( $\$ 1626 \mathrm{Abs}$. $2 \mathrm{BCB}$ ). Die Beachtung dieser „Wohlschranke“ bemisst sich vor allem nach dem Grundsatz der Verhältnismäßigkeit, wobei der staatlichen Gemeinschaft nur eine Wächterfunktion zukommt.

\subsubsection{Patientenverfügungen und einwilligungsfähige Minderjährige}

Wenn ein ehemals einwilligungsfähiger Erwachsener in einer momentanen Lebenslage nicht mehr fähig ist, seinen Willen zu bilden oder zu äußern, müssen die behandelnden Ärzte und sein gesetzlicher Vertreter trotzdem - soweit möglich - sein Recht auf Selbstbestimmung wahren, wie dies zum Beispiel durch die Heranziehung einer zuvor erstellten Patientenverfügung geschieht. Der darin niedergelegte Wille bzw. die darin festgelegten Behandlungswünsche müssen beachtet werden. Indem der volljährige Patient in einwilligungsfähiger Situation seinen Willen schriftlich fixiert, nutzt er die Möglichkeit, seiner Souveränität auch im Zustand einer zukünftigen Einwilligungsunfähigkeit Geltung zu verschaffen.

Für einen minderjährigen, aber einwilligungsfähigen Patienten existiert dieses Mittel zur Wahrung seiner Autonomie in Deutschland derzeit nicht. Die rechtlichen Regelungen zur Patientenverfügung sind vor allem in $\mathbb{1}$ 1901a BGB (Bürgerliches Gesetzbuch) und damit im Betreuungsrecht angesiedelt. Voraussetzung für die Erstellung einer Patientenverfügung ist daher neben der Einwilligungsfähigkeit auch die Volljährigkeit des Verfügenden. Darin liegt eine Benachteiligung einwilligungsfähiger, aber noch minderjähriger Patienten gegenüber Patienten, die das 18. Lebensjahr bereits vollendet haben. Dass der aktuell ausgedrückte Wille eines einwilligungsfähigen Jugendlichen für den Arzt rechtsverbindlich ist, nicht aber sein prospektiver (verfügter) Wille, überzeugt normativ nicht.

Die von der Deutschen Akademie für Kinder- und Jugendmedizin e.V. (DAKJ) geforderte diesbezügliche Korrektur der Rechtslage ist daher zu unterstützen (DAKJ 2014). Zurzeit ist eine denkbare Alternative zur Erstellung einer Patientenverfügung die Ab- 
fassung einer antizipativen Behandlungsverfügung, die vom minderjährigen Patienten und seinen Eltern gemeinsam erstellt und unterzeichnet wird. Ein solches Dokument erlangt aber nicht die rechtliche Verbindlichkeit einer Patientenverfügung.

Eine antizipative Behandlungsvereinbarung, die von dem minderjährigen, aber einsichtsfähigen Patienten gemeinsam mit seinen Eltern erstellt wird, hat nicht die rechtliche Verbindlichkeit einer Patientenverfügung, kann aber den Willen des Minderjährigen gut abbilden und behandlungsleitend sein.

\subsection{Der notfallmedizinische Einsatz und besondere pädiatrische Notfallsituationen}

Der Tod von Kindern und Jugendlichen (o-17 Jahre) ist zu einer Seltenheit geworden, obwohl diese in unserer Gesellschaft etwa 20\% der Bevölkerung stellen. Weniger als $2 \%$ aller Todesfälle entfallen auf diese Altersgruppe (AAP 2014). Noch vor einem Jahrhundert stellten die Todesfälle von Kindern unter fünf Jahren dagegen noch etwa 30\% aller Todesfälle. Diese Zahlen belegen, wie selten der Tod eines Kindes sich heutzutage in westlichen Industrienationen ereignet und wie sehr er zu einer singulären Tragödie und einem niemals erwarteten Ereignis geworden ist, anders als der Tod älterer Angehöriger. An großen pädiatrischen Notfallaufnahmen ereignet sich der Tod eines Kindes nur etwa bei einer von 15.000 Notfallkonsultationen (AAP 2014). Dies zeigt aber auch, wie selten ein solches Ereignis für jeden professionellen Helfer, selbst für den vornehmlich pädiatrisch Arbeitenden geworden ist. Gleichzeitig ist die Zahl von Notfallmedizinern mit einer großen Erfahrung im Umgang mit Todesfällen in dieser Altersgruppe ebenfalls klein.

Jede notfallmedizinische Versorgung stellt hohe Anforderungen an alle Beteiligten. Nach besonders belastenden Einsätzen ist eine nachbereitende Besprechung des Ablaufes in nahem zeitlichem Abstand im multidisziplinären Kreis der beteiligten Helfer sinnvoll. Hier sollten die lückenlose Dokumentation der Abläufe sowie die Bewertung derselben und die Rolle jedes Beteiligten in der Rückschau besprochen und gewürdigt werden. Diese Gespräche, gegebenenfalls im Rahmen einer Supervision, dienen der persönlichen Verarbeitung, erhöhen die Identifikation jedes Einzelnen mit dem Team und tragen zu einem strukturierteren Ablauf zukünftiger Notfalleinsätze bei.

\subsubsection{Geburt und Frühgeburtlichkeit}

Zwar erfolgt in Deutschland, anders als in anderen Nachbarstaaten (z.B. in den Niederlanden), die bei weitem überwiegende Zahl aller Geburten im Krankenhaus, aber gelegentlich wird auch ein Notfallteam mit einer Geburtssituation außerhalb der Klinik konfrontiert. Das sind neben plötzlichen Gefährdungssituationen bei geplanten Hausgeburten auch rasch eintretende Geburtsbestrebungen bei bekannten, meist gut kontrollierten Schwangerschaften. Zum anderen gibt es in seltenen Fällen Schwangere, die gegenüber ihrer Umwelt die Schwangerschaft verbergen oder diese tatsächlich gänzlich nicht bemerkten, sodass die Frau dann plötzlich und unvorbereitet in eine Geburtssituation gerät. 
Eine komplikationslose Geburt ist für alle Beteiligten ein freudiges Ereignis, wenn die Natur ihren Verlauf von alleine nimmt. Dann bedarf das Neugeborene vor allem der mütterlichen Wärme in direktem Hautkontakt und beide einer weiteren fachgerechten Betreuung in der nächstgelegenen Geburtshilfe. Ist die Geburt als solche aber noch nicht beendet, ergibt sich für alle meist völlig untrainierten Beteiligten eine potenziell gefährliche Sachlage, der man so effektiv wie möglich mit einer Verzögerung des Geburtsverlaufes entgegentreten sollte.

Immer ist in dieser Situation, in der außerklinisch eine genauere Zustandsbeurteilung des Feten nicht möglich ist, der intrauterine Transport des ungeborenen Kindes in die nächstgelegene geburtshilfliche Klinik zu bevorzugen. Von hier aus wird nach gynäkologischer Bewertung des Geburtsverlaufes gegebenenfalls der sekundäre intrauterine Transport in das zugeordnete Perinatalzentrum veranlasst oder das neonatologische Notfallteam bei nicht mehr zu verhindernder Geburt in diese Geburtsklinik hinzugerufen.

Wenn die Geburt außerklinisch nicht zu verhindern ist, hat der Notarzt fortan zwei Patienten, um die er sich kümmern muss. Er sollte deshalb auch mit den Grundzügen der neonatalen Adaptation, ihren Störungen und auch mit den Grundzügen der neonatalen Reanimation vertraut sein. In der extremen Situation einer unmittelbar vitalen Bedrohung von Mutter und Kind (z.B. atone Uterusblutung und Asphyxie des Neugeborenen) gilt möglicherweise aus anthropologischen und moralischen Gründen das primäre Reanimationsbestreben der Mutter, nicht aber aus rechtlichen, denn hier besteht eine Gleichwertigkeit menschlichen Lebens, was dem Arzt von Rechts wegen die Entscheidung überlässt, welche der kollidierenden Pflichten gleichen Ranges er erfüllt.

Eine sekundäre Hinzuziehung des regional zuständigen neonatalen Notarztteams sollte in jedem Falle und so früh wie möglich erfolgen, wenn die Geburt im außerklinischen Kontext nicht mehr verhinderbar ist. Bis zum Eintreffen des Neonatologen erfolgt die Unterstützung des Neugeborenen nach den Richtlinien des Newborn Life Support (NLS). Eine Entscheidung über den Abbruch nicht erfolgreicher Reanimationsmaßnahmen sollte nur mit dem sekundär hinzugerufenen neonatologischen Notfallteam gemeinsam gefällt werden.

Nur so ist größtmögliche Sicherheit gegeben, dass eine sachgerechte Versorgung bei jeder Bedrohungssituation von der Frühgeburtlichkeit, über den Umgang mit einer kindlichen Asphyxie bis hin zur Versorgung sich abzeichnender Erkrankungen oder eventuell vorhandener Fehlbildungen stattfindet und damit das Notfallteam insgesamt von späteren inneren oder äußeren Vorwürfen geschützt ist.

Mit der besonderen Versorgungssituation von extrem unreifen „Frühgeborenen an der Grenze der Lebensfähigkeit “ befasst sich auch die neue Leitlinie der Arbeitsgemeinschaft wissenschaftlich medizinischer Fachgesellschaften (AWMF), die die Beratung mit den Eltern in den Vordergrund stellt (AWMF 2014). Dieses „shared decision making“ setzt eine enge Einbindung der Eltern voraus, die „in interdisziplinären Beratungsgesprächen in die dann gemeinsam zu findende und zu tragende Entscheidung“ einbezogen werden. Dies erfolgt „,bei veränderter Befundlage auch in kurzen Abständen wiederholt“ und ist „nach Teilnehmern, Zeitpunkt und Inhalt zu dokumentieren“.

In der Klinik wird das unreife Frühgeborene jedoch zum ethischen Notfall, wenn nicht im Vorfeld diese multidisziplinären Gespräche mit den Eltern zur Verabredung einer klaren Vorgehensweise unter und nach der Geburt geführt worden sind. 


\begin{abstract}
Besonders am Beginn des Lebens kann es zu dramatischen Ereignissen kommen. Wenn der Gesundheitszustand des Kindes das Auftreten von peripartalen Komplikationen vermuten lässt, ist es wichtig, dass sich das Behandlungsteam gemeinsam mit den Eltern rechtzeitig über mögliche Grenzen in der Therapie verständigt.
\end{abstract}

\title{
10.6.2 Plötzlicher Kindstod
}

Der plötzliche Kindstod (sudden infant death syndrome [SIDS]) ist noch immer nicht ursächlich erklärbar. Dieses Phänomen ist aber zumindest ätiologisch eingrenzbar und stellt eine Ausschlussdiagnose dar, die sich erst nach sorgfältiger Anamnese und gründlicher postmortaler Untersuchung, die keine andere diagnostizierbare Todesursache zeigt, stellen lässt (Jorch $u$. Poets 2014). Durch intensive epidemiologische Studien konnten in den letzten Jahrzehnten einige prägnante Risikofaktoren identifiziert werden. Groß angelegte Präventionsmaßnahmen mit mehreren nationalen und internationalen Aufklärungskampagnen zu Säuglingspflegepraktiken waren darüber hinaus geeignet, die Inzidenz von SIDS in vielen westlichen Nationen um mehr als 50-70\% zurückzudrängen. Die Diagnose führt aber noch immer die Liste der Todesursachen von Kindern jenseits der Neugeborenenperiode vor den Unfällen im Verkehr und Haushalt und malignen und selten infektiösen Erkrankungen an.

Wenn ein Notarztteam zu einem solchen Vorfall alarmiert wird, ist der Tod des Patienten meist schon vor längerer Zeit eingetreten. Wenn die Eltern oder andere eine Laienreanimation begonnen haben, wird das Team diese als solche zunächst einmal übernehmen und fortführen. Lassen sich hingegen schon primär oder im Verlauf einer zunächst begonnenen Reanimation sichere Zeichen des schon eingetretenen Todes erkennen, wird das Team vor Ort den eingetretenen Tod bereits am Ereignisort bescheinigen.

Allein aus dem Fehlen von Return Of Spontaneous Circulation (ROSC) darf aber andererseits auch unter laufenden Reanimationsbemühungen nicht immer zweifelsfrei der schon unumkehrbar eingetretene Tod konstatiert werden. Es ist daher völlig legitim, auch in dieser Situation sekundär, über die Feuerwehrleitstelle, das pädiatrische Notfallteam zu einer gemeinsamen Entscheidung hinzuzuziehen oder bei größeren Distanzen einen Transport unter Fortführung einer kardiopulmonalen Reanimation (Cardiopulmonary Resuscitation $[\mathrm{CPR}]$ ) in die nächstgelegene klinische Notaufnahme vorzunehmen. Dies gilt umso mehr, je unerfahrener das Team vor Ort mit dieser Situation ist. In Ländern, in denen zu großen Teilen die primäre Rettung von paramedizinischen Diensten vorgenommen wird, trifft man häufiger auf ein solches Vorgehen, weil sich diese Rettungsteams mit der Feststellung des Todes bei einem Kind noch schwerer tun als bei einem Erwachsenen (AAP 2014). Im notärztlich strukturierten deutschen Rettungswesen wird überwiegend der eingetretene Tod bereits im häuslichen Kontext attestiert und mit Reanimationsmaßnahmen gar nicht erst begonnen.

Es kommt deshalb schon dem primären Notfallteam unter Zuhilfenahme der psychosozialen Notfallversorgung (PSNV) die Aufgabe zu, den Eltern diese Todesnachricht $\mathrm{zu}$ vermitteln. Hierbei sind eine klare, ruhige Sprache wichtig sowie die eindeutige 
Erklärung, dass das Kind tot ist. Die Form dieser Vermittlung der Todesnachricht ist extrem wichtig und sollte im Bewusstsein erfolgen, dass die Eltern in all ihrer Ohnmacht diese Situation nie vergessen werden.

In der sorgfältigen Dokumentation kommt der gründlichen Anamnese und der Auffindungssituation eine große Bedeutung zu, bildet sie doch mit der sich dann notwendigerweise anschließenden Obduktion die Grundlage für die Einordnung dieses Todes als SIDS.

Das Notfallteam sollte mit ruhigen und vermittelnden Worten den Eltern auch die erforderliche Ermittlung durch die Polizei nahe bringen. Es ist wichtig zu betonen, dass dies nicht Ausdruck irgendeines Misstrauens ist, sondern ebenso zu ihrer Entlastung geschieht, wie die in Deutschland bei unklaren Todesfällen in den ersten zwei Lebensjahren von der zuständigen Staatsanwaltschaft wohl immer angeordnete Obduktion des Kindes.

Es sollten die Eltern unbedingt Gelegenheit bekommen, sich von ihrem verstorbenen Kind verabschieden zu können, auch wenn der Leichnam von der Polizei in Beschlag genommen wird.

Zur unmittelbaren Mitbetreuung dieser Verlustsituation sollten der zuständige Kinderarzt und der Hausarzt der Familie informiert werden. Diese können in der Nachbetreuung nach Abschluss des Ermittlungsverfahrens dann das rechtsmedizinische Gutachten anfordern und mit den Eltern dieses Ergebnis besprechen, um ihnen abschließend alle Schuldgefühle nehmen zu können.

Schließlich sollte den Eltern rasch eine Kontaktaufnahme mit anderen betroffenen Eltern, z.B. über die Selbsthilfegruppe Gesellschaft zur Erforschung des plötzlichen Säuglingstodes (GEPS, Elterninitiative plötzlicher Säuglingstod), ermöglicht werden.

Dem retrospektiven Teamgespräch im multidisziplinären Helferkreis kommt eine zentrale Bedeutung in der langfristigen und nachhaltigen Verarbeitung einer Notfallsituation mit Blick auf die emotionale Stabilität jedes Einzelnen und die kontinuierliche Verbesserung des zukünftigen Prozessablaufes zu.

\subsubsection{Misshandlung, Missbrauch, Vernachlässigung}

Zählt man alle Formen von körperlicher, sexueller und seelischer Kindesmisshandlung zusammen, dann erfahren nach Schätzungen möglicherweise 10\% aller Kinder eine solche Form meist wiederholter Gewalt, zumeist durch nahe Angehörige. Das Recht auf eine gewaltfreie Erziehung ist jedoch seit 2000 gesetzlich im Bürgerlichen Gesetzbuch verankert ( $\$ 1631$ Abs. 2 BGB).

Gerade im notfallmedizinischen Einsatz werden Kinder immer wieder mit Verletzungen vorgestellt, die auf unerklärlichem Wege eingetreten sind oder für die völlig unstimmige oder rasch wechselnde Erklärungen angeführt werden. Es sind vor allem Kinder unter vier Jahren, die hierbei das höchste Risiko für tödliche Misshandlungen tragen.

Es sollten alle im Notfallwesen arbeitenden Mitarbeiter über anamnestische Besonderheiten und körperliche Untersuchungsbefunde bei Misshandlungen ausgebildet 
sein. Diese bilden häufig die Grundlage für eine Anfangsvermutung, der durch eine sorgfältige Anamnese und eine eingehende vollständige körperliche Untersuchung nachgegangen werden sollte. Wenn Verletzungen bei einem Säugling oder Kleinkind beobachtbar sind, die sich dieses schlechterdings nicht selbst zugefügt haben kann, wie z.B. symmetrische strumpfartige Verbrühungen an den Extremitäten, kreisrunde Brandwunden durch ausgedrückte Zigaretten auf der Haut des Kindes oder Hämatome als Negativabdruck einer Hand, gilt es, diese Alarmzeichen ernst zu nehmen. Häufig findet sich eine inadäquat lange Latenz zwischen Verletzung und erster klinischer Vorstellung. Gelegentlich werden bei der detaillierten klinischen Untersuchung weitere Verletzungszeichen entdeckt. Auch werden häufig unterschiedliche Ärzte aufgesucht („doctor hopping“).

Eventuell sind zudem erkennbare Belastungen der Partnerschaftsbeziehung und der Familie oder weitere Anzeichen häuslicher Gewalt erkennbar.

Bei geringstem Verdacht auf das Vorliegen einer Verletzung des Kindes durch Dritte sollte eine stationäre Aufnahme erfolgen, um diesem Hinweis in Ruhe nachgehen zu können.

Hierbei wird es ratsam sein, diese Mutmaßung nicht gleich offen zu legen, da sie doch, kaum geäußert, gleich zu elterlicher Abwehr führen wird. In einer solchen Situation sollte einem elterlichen Wunsch auf eine lediglich ambulante Versorgung, die mit einer Verweigerung des Transportes in die nächstgelegene Kindernotfallaufnahme einhergeht, deeskalierend, aber bestimmt entgegen getreten werden.

In der Klinik erfolgt dann ein definiertes Untersuchungsprogramm zum Screening auf mögliche äußere Gewalteinwirkungen. Dieses schließt laborchemische, technische und strukturgebende, aber auch psychosoziale Untersuchungen ein. Ziel ist dabei weniger, eine Anklage und justiziable Verfolgung des vermeintlichen Täters zu erreichen, als vielmehr zum Wohle des Kindes ein soziales Betreuungsnetzwerk zur Vermeidung weiterer Gewalteinwirkung in multiprofessioneller Kooperation unter Beteiligung des Jugendamtes, der Kinderschutzgruppen, aufsuchender familienentlastender Sozialdienste und des weiterbetreuenden Kinderarztes zu knüpfen.

\section{Schon der leiseste Verdacht auf eine Verletzung durch fremde Hand sollte im Notdienst immer zur stationären Einweisung führen.}

Es ergibt sich zwangsläufig ein Konflikt zwischen der „ärztlichen Schweigepflicht“ ( $\$ 203$ StCB) und einer „Offenbarungsbefugnis“ aus dem „rechtfertigenden Notstand“ nach $\mathbb{3} 34 \mathrm{StGB}$, wobei die Kindesgesundheit als vorrangiges Interesse anzusehen ist, wenn die ernsthafte Gefahr weiterer Gewaltaussetzung besteht. Hierzu enthält seit 2012 das Gesetz zur Kooperation und Information im Kinderschutz (KKG) spezielle Regelungen. Auch die beruflich befassten Ärzte sollen bei gewichtigen Anhaltspunkten für eine Kindeswohlgefährdung die Situation mit dem Kind oder Jugendlichen und den Personensorgeberechtigten erörtern ( $\$ 4$ Abs. 1 KKG). Scheidet das zur Gefahrabwendung aus und wird die Einschaltung des Jugendamtes für erforderlich gehalten, darf dieses trotz Schweigepflicht informiert werden ( $\$ 4 \mathrm{Abs} .3$ $\mathrm{KKG}$ ). Auf den eigentlich obligaten Hinweis darauf an die Betroffenen kann verzichtet werden, wenn ansonsten der wirksame Schutz des Kindes oder Jugendlichen infrage gestellt ist. 
Meist ist es hilfreich, wenn eine Strafanzeige durch das auf diese Weise einbezogene Jugendamt als der ordnungsrechtlichen Behörde und nicht von den um Vermittlung bemühten primär medizinisch Verantwortlichen vorgenommen wird. Auch wenn bei einer solchen Straftatermittlung eine rechtskräftige Verurteilung häufig nicht zu erreichen ist, so kann diese juristische Intervention doch die elterliche Kooperationsbereitschaft erhöhen und stellt auch die Grundlage für eine familiengerichtliche Bewertung dar.

Den Ersthelfern kommt bereits in der Erfassung des Problems häufig eine Schlüsselrolle zu, da sie doch die Einzigen sind, die schon primär im häuslichen Kontext der Familie tätig werden.

Werden eine vital erforderliche erste Diagnostik und Therapie, gegebenenfalls schon der Transport in die nächstgelegene Notfallambulanz durch die Eltern abgelehnt, muss das Notfallteam im Ausnahmefall die Ordnungskraft der Polizei in Anspruch nehmen. Eine solche Konfrontation gilt es möglichst zu vermeiden und durch Benennung der bestehenden Gefährdung eine hinreichende Einsicht zu erzielen. Bei jedem Hinweis auf eine Situation, in der das Kind durch seine Eltern gefährdet sein könnte, sollte ein Transport in die zugeordnete Kinderklinik erfolgen, um hier auch der Frage nach einer Kindeswohlgefährdung durch mögliche Misshandlung oder Vernachlässigung nachgehen zu können. Ist eine offenkundige Unfallsituation gegeben, sollte die Polizei für eine Unfallaufnahme hinzugezogen werden. Dies gilt insbesondere immer dann, wenn es sich um schwere Verletzungen handelt oder das offenkundige Verletzungsbild und ein geschilderter Unfallhergang nicht zueinanderpassen. Auch alkoholisierte oder anderweitig unkontrollierte Eltern oder Betreuungspersonen erfordern gelegentlich die Anforderung polizeilicher Unterstützung.

\subsection{Anwesenheit der Eltern bei Reanimationsvorgängen}

Säuglinge und Kleinkinder befinden sich in aller Regel in Begleitung eines Elternteils, dem die notwendigen Maßnahmen parallel zur Akutversorgung erläutert werden können. Die Eltern sind dabei unabhängig von ihrem religiösen, weltanschaulichen und kulturellen Hintergrund in einer für sie maximalen Ausnahmesituation in großer Sorge um ihr Kind und deshalb nicht immer rationalen Überlegungen zugänglich.

Aus den genannten Umständen kommt der Besprechung verschiedener medizinischer, wie psychosozialer Besonderheiten und der Vorbereitung auf eine solche Situation eine große Bedeutung zu.

Wenn Kinder in Not geraten, geschieht dies häufig in Anwesenheit der Eltern. Die Beantwortung folgender Fragen ist unter diesen Umständen besonders dringlich:

- Wie können Angehörige in einer solchen Situation mit eingebunden werden?

- Wie können Reanimationsabläufe unter der engen Beobachtung der Eltern gesteuert werden?

- Wann dürfen Reanimationsbemühungen beendet werden?

- Wie kann in einer offenkundig palliativen Versorgungssituation die Reanimation eines Patienten, der wegen seiner Grunderkrankung nicht wiederbelebt werden sollte, vermieden werden?

- Wie kann mit eigenen emotionalen Implikationen, die eine solche Reanimation nach sich zieht, auch im Team umgegangen werden? 
Jedem, der eine Sterbenssituation mit begleitet, sollte bewusst sein, dass die Angehörigen die Form, Intensität und den Inhalt der Versorgung bis an das Ende ihres Lebens nicht vergessen werden. Deshalb besteht eine besondere Fürsorgepflicht auch gegenüber den hoch belasteten Eltern.

Viele Notfallmitarbeiter stehen der Anwesenheit von Eltern oder anderen nahen Bezugspersonen bei den erforderlichen medizinischen Versorgungsmaßnahmen im Rahmen einer Notfallsituation (Reanimation) immer noch sehr kritisch gegenüber, sodass vielfach die erste Maßnahme bei Eintreffen in eine außer-oder innerklinische Notsituation darin besteht, diese Angehörigen aus dem Raum zu begleiten. Der initiale Impuls, Eltern an solchen Situationen nicht teilhaben zu lassen, bei denen mit einer vollständigen Erholung des Patienten nicht gerechnet werden kann, ist in der Furcht begründet, dass die Maßnahmen eben nicht erfolgreich verlaufen könnten und die Angehörigen so durch das Zeugnis der erfolglosen Reanimation eine noch größere Belastung erfahren. Sicher bestehen auch Bedenken hinsichtlich der rechtlichen Ansprüche gegenüber vermeintlich erfolgten „Fehlern“, ebenso wie die Angst, dass die professionellen Notfallhelfer von der Durchführung effektiver Reanimationsbemühungen abgelenkt werden.

Hierzu gibt es im deutschen Kontext bislang keine wissenschaftliche Aufarbeitung. Einzelne amerikanische und schwedische Arbeiten zeigen, dass keine Unterbrechung der Reanimationsbemühungen oder eine Verschlechterung ihrer Ergebnisse durch die Anwesenheit Dritter beobachtet wurde. 95\% der befragten Familienmitglieder bekundeten, dass sie wieder wünschen würden, bei einer solchen Situation anwesend sein zu dürfen, und dass es ihnen zur Verarbeitung hilfreich war (AAP 2014).

Neuere Studien und Berichte reflektieren auch eine wachsende Kompetenz von Notfallmitarbeitern im Umgang mit der Präsenz von Familienmitgliedern bei solchen Reanimationsbemühungen. Dieser Umstand ist regelmäßig Gegenstand der EPLS Kurse in der pädiatrischen Reanimationsausbildung nach den Richtlinien des European Resuscitation Council (ERC). Zweifellos erscheint es dafür aber notwendig, explizit benannte und in dieser Art von Begleitung erfahrene Mitarbeiter den Eltern zur Seite zu stellen. Im außerklinischen Kontext muss so die Zeit bis zum Eintreffen des psychosozialen Notfallbegleitungsteams (PSNV) überbrückt werden. Innerhalb der Klinik werden hier die Mitarbeiter der Seelsorge, der klinischen Psychologie oder die in der Betreuung der betroffenen Eltern bereits im Vorfeld involvierten Mitarbeiter die größte Unterstützung bieten können.

In der aktuellen Literatur wird die Anwesenheit der Eltern somit weitestgehend unterstützt, sodass dies auch in der curricularen Rettungsausbildung einen eigenen Stellenwert bekommen hat.

Die Versorgung eines sterbenden Kindes muss immer auch die Versorgung und Begleitung der Eltern beinhalten und berücksichtigen.

\subsection{Tod und Abschied}

Wenngleich zu Beginn einer Notfallversorgung immer der Versuch zum Lebenserhalt des Kindes im Vordergrund steht, ergibt sich bei einer dramatisch verlaufenden 
neurologischen Verschlechterung bis hin zum irreversiblen Hirnfunktionsausfall für die weitere Intensivtherapie auch der Gedanke an eine mögliche Organspende. Dieser Gedanke muss nach Feststellung des Hirntodes aufgegriffen werden und kann manchen Eltern angesichts des plötzlichen Verlusts ihres Kindes ein gewisser Trost sein.

Die elterliche Präsenz beim Reanimationsvorgehen wurde mit ihrem Für und Wider vorangehend besprochen. Dass die Anwesenheit der Eltern in dem Moment des Todeseintritts eine herausragende Bedeutung besitzt, wird wiederum schon sehr viel länger akzeptiert. Dies ist im intensivmedizinischen Arbeitsfeld regelmäßig präsent. Bei einer begonnenen, in ihrem weiteren Verlauf aber eher diskussionswürdigen Reanimation erscheint es akzeptabel, diese auch bis zum Zeitpunkt des Eintreffens der Eltern fortzusetzen. Ihnen die Anwesenheit in diesem letzten für sie so wichtigen Lebensmoment zu ermöglichen, sollte immer zu gewährleisten versucht werden. Hier spätestens sollte in einem primär christlich geprägten kulturellen Umfeld die Frage nach einer Nottaufe, Krankensalbung oder anderen religiösen Handlungen gestellt werden.

Wenn wir von diesem Vorgehen abweichen müssen, weil der Zeitraum aus verschiedenen Gründen zu lang erscheint, ist es den Eltern meist ein großer Trost, wenn ihnen versichert werden kann, dass ihr Kind keine Qualen erleiden musste und auch im Tod nicht allein war. Bestenfalls sollte auch das verstorbene Kind bis zum Eintreffen der Eltern nicht allein gelassen werden.

Selbstverständlich brauchen Eltern dann hinreichend Zeit, um von ihrem Kind Abschied zu nehmen. Hierfür sind stationseigene Verfahrensempfehlungen sinnvoll, die als schriftlich definierte Standards hinterlegt werden sollten. Neben den Standard Operation Procedures (SOPs) einzelner Abteilungen gibt es in Deutschland dazu bislang wenig eigene Forschungsergebnisse oder gesellschaftsweite Empfehlungen, sodass die von dem Committee on Bioethics innerhalb der AAP erarbeiteten policy statements und consensus guidelines auch im europäischen Kontext hilfreich sind (AAP 2014).

Es hat sich bewährt, einen angemessen ausgestatteten Raum bereitzuhalten, in dem das verstorbene Kind nach der Beendigung der medizinisch-pflegerischen Maßnahmen aufgebahrt wird. Unterschiedlichsten Ritualen von der Waschung, Herrichtung des Leichnams, Bekleidung bis zur Beigabe von Kuscheltieren oder Ähnlichem ist Rechnung zu tragen. Hand- und Fußabdrücke oder auch ein Foto können trostspendend sein. Den Eltern und auch einem größeren Kreis von Freunden und Angehörigen, vor allem unter Einbeziehung der Geschwisterkinder, sollte möglichst so viel Zeit und Raum angeboten werden, wie sie es für sich wünschen.

\section{Literatur}

AAP American Academy of Pediatrics (2011) Policy Statement-Consent for Emergency Medical Services for Children and Adolescents. Pediatrics 128: 427-433

AAP American Academy of Pediatrics (2014) Technical Report-Death of a Child in the Emergency Department. Pediatrics 134: e313-e330

Adams HA, Flemming A (2015) Analgesie, Sedierung und Anästhesie in der Notfallmedizin. Anästh Intensivmed 56: 75-90

Arbeitsgemeinschaft wissenschaftlich medizinischer Fachgesellschaften (AWM) Leitlinie (2014) Frühgeborene an der Grenze der Lebensfähigkeit, AWMF Leitlinien Register Nr. 024/19. Letzte Überarbeitung 04/2014, Gesellschaft für Neontologie und pädiatrische Intensivmedizin 
Beauchamp T, Childress I (2012) Principles of biomedical ethics. $7^{\text {th }}$ edition. Oxford University Press, Oxford, New York, Toronto

BGH NJW 1959, 811, „Einwilligung Minderjähriger in ärztliche Heilbehandlung“

Deutsche Akademie für Kinder- und Jugendmedizin DAKJ (2009) Kommission für ethische Fragen: Positionspapier zur Begrenzung lebenserhaltender Therapie im Kindes- und Jugendalter. Monatsschr Kinderheilkd 157: 43-48

Deutsche Akademie für Kinder- und Jugendmedizin DAKJ (2014) Stellungnahme zu Patientenverfügungen von Minderjährigen. www.dakj.de/media/stellungnahmen/ethische-fragen/2014-patientenverfuegung-minderjaehrige.pdf (Zugriff am 17.04.2015)

Dierks C, Graf Baumann T, Lenard H (1995) Therapieverweigerung bei Kindern und Jugendlichen Medizinrechtliche Aspekte 6. Einbecker Workshop der dt. Gesellschaft für Medizinrecht im März 1995. Springer, Berlin

Führer M, Duroux A, Jox R, Borasio G (2009) Entscheidungen am Lebensende in der Kinderpalliativmedizin. Monatsschr Kinderheilkd 157: 18-25

Gillis I (2008) „We want everything done“. Arch Dis Child 93: 192-193

Harder Yv (2004) Rechtliche Besonderheiten bei der Behandlung minderjähriger Patienten. Anästhesist 53: $1105-1110$

Herrmann B, Noeker M, Franke I (2014) Misshandlung, Missbrauch und Vernachlässigung von Kindern. In: Hoffmann G, Lentze M, Spranger I, Zepp F (Hrsg.) Pädiatrie. Springer, Berlin, S. 169-177

Jorch G, Poets C (2014) Der plötzliche Kindstod. In: Hoffmann G, Lentze M, Spranger J, Zepp F (Hrsg.) Pädiatrie. Springer, Berlin, S. 163-169

Jox R, Führer M, Borasio G (2009) Patientenverfügung und Elternverfügung. Monatsschr Kinderheilkd 157: 26-32

Kessler-Weinrich A (2015) „Zum Wohl des Kindes!?“ Die Bedeutung der Eltern im Entscheidungsfindungsprozess am Beispiel ethischer Fallgespräche, Abschlussarbeit MA, Mainz

Kobert K, Löbbing T (2013) Erfahrungsbericht zu Ethikvisiten und ethischen Liaisondiensten als Elemente der Ethikberatung. In: Steger F (Hrsg.) Klinische Ethikberatung. mentis Verlag, Münster, S. 113-126

Niethammer D (2014) Ethik in der Pädiatrie. In: Hoffmann G, Lentze M, Spranger J, Zepp F (Hrsg.) Pädiatrie. Springer, Berlin, S. 4-7

Nikendei A (2012) Begleitung von Kindern und Jugendlichen. In: Nikendei A (Hrsg.) Psychosoziale Notfallversorgung (PSNV). Stumpf+Kossendey Verlag, Edewecht, S. 315-330

Putz W, Steldinger B (2009) Rechtliche Aspekte der Therapiebegrenzung in der Pädiatrie. Monatsschr Kinderheilkd 157: 33-37

Rellensmann G, Hasan C (2009) Empfehlungen zum Vorgehen in Notfallsituationen. Monatsschr Kinderheilkd 157: 38-42

Royal College of Paediatrics and Child Health (2004) Witholding or withdrawing life sustaining treatment in children: A framework for practice. http://www.rcpch.ac.uk/system/files/protected/page/Witholding\%20.... pdf (Zugriff am 27.04.2015)

Spitzer P, Höllwarth M (2014) Unfälle und Unfallverhütung. In: Hoffmann G, Lentze M, Spranger J, Zepp F (Hrsg.) Pädiatrie. Springer, Berlin, S. 178-183

Steurer M, Berger T (2012) Spezifische ethische Konflikte in der pädiatrischen und neonatologischen Intensivmedizin. In: Salomon F (Hrsg.) Praxisbuch Ethik in der Intensivmedizin. 2. Auflg. Med. Wiss. Verlagsges. Berlin, S. 163-182

\section{Abkürzungsverzeichnis}

AAP American Academy of Pediatrics

ATLS Advanced Trauma Life Support

AWMF Arbeitsgemeinschaft wissenschaftlich medizinischer Fachgesellschaften

BGB Bürgerliches Gesetzbuch

CPR Cardiopulmonary Resuscitation

DAKJ Deutsche Akademie für Kinder- und Jugendmedizin

EPLS European Pediatric Life Support 


$\begin{array}{ll}\text { ERC } & \text { European Resuscitation Council } \\ \text { GEPS } & \text { Gesellschaft zur Erforschung des plötzlichen Säuglingstodes } \\ \text { GG } & \text { Grundgesetz } \\ \text { KKG } & \text { Gesetz zur Kooperation und Information im Kinderschutz } \\ \text { NLS } & \text { Newborn Life Support } \\ \text { PSNV } & \text { psychosoziale Notfallversorgung } \\ \text { ROSC } & \text { Return Of Spontaneous Circulation } \\ \text { SOP } & \text { Standard Operation Procedure } \\ \text { StGB } & \text { Strafgesetzbuch }\end{array}$

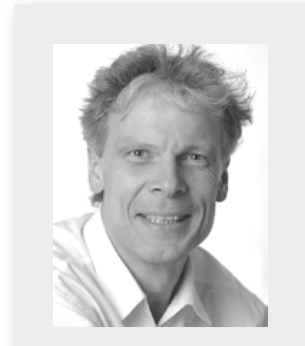

Dr. med. Thomas M. Boesing

Studium der Humanmedizin in Münster und Dublin. Ausbildung zum Facharzt für Kinder- und Jugendmedizin in Bielefeld, Weiterbildung Neonatologie und pädiatrische Intensivmedizin in Frankfurt/M, Weiterbildung pädiatrische Pneumologie und pädiatrische Gastroenterologie in Bielefeld, Fernstudium zum Ethikberater im Gesundheitswesen an der Uni Nürnberg und Basel, Leitender Arzt Abteilung Neonatologie und pädiatrische Intensivmedizin Kinderzentrum Bielefeld-Bethel am Evangelischen Krankenhaus Bielefeld, Mitglied im Klinischen Ethik-Komitee seit dessen Bestehen 2003.

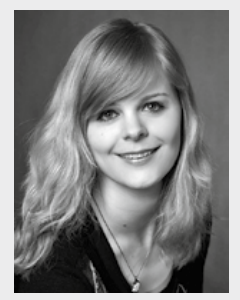

\section{Tanja Löbbing, Master of Medicine, Ethics and Law}

Studium der Gesundheitswissenschaften (Health Communication) in Bielefeld, Masterstudium Medizin-Ethik-Recht in Halle an der Saale, Trainerin für Ethikberatung (AEM). Seit 2012 Tätigkeit als Klinische Ethikerin im Ev. Krankenhaus Bielefeld, Mitglied im Klinischen Ethik-Komitee.

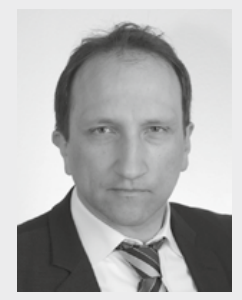

\section{Prof. Dr. jur. Bernhard Kretschmer}

Studium der Rechtswissenschaft in Bielefeld, Referendariat am Oberlandesgericht Hamm, Lehr- und Forschungsaufgaben an den Universitäten Bielefeld, München, Bochum und Gießen, Habilitation 2010, Inhaber eines Lehrstuhls für Straf- und Strafprozessrecht mit medizinstrafrechtlichem Schwerpunkt: zunächst von 2011 bis 2013 an der Ruhr-Universität Bochum, seither an der Justus-Liebig-Universität Gießen; Mitglied der Ethik-Kommissionen der dortigen Fachbereiche für Medizin und Psychologie, Mitglied in den Klinischen EthikKomitees des Evangelischen Krankenhauses Bielefeld und des Markus-Krankenhauses Frankfurt a.M. 


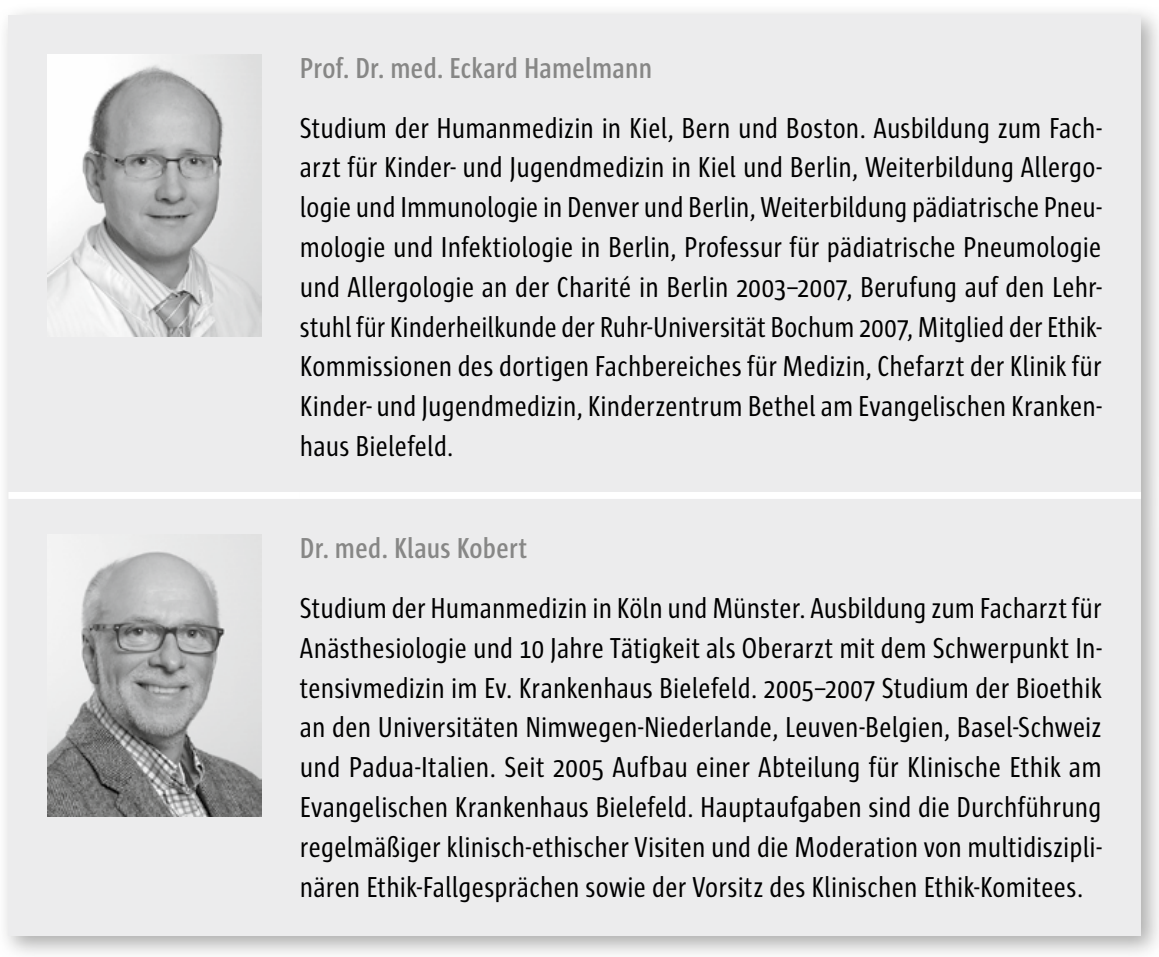

